\title{
BALTASAR DE LOS REYES MARRERO (1752-1809): PRIMER EDUCADOR DE LA ENSEÑANZA DE LA FÍSICA MODERNA EN LA UNIVERSIDAD DE CARACAS (ÚLTIMAS DÉCADAS DEL SIGLO XVIII) (PARA UNA HISTORIA CONECTADA DE LA HISTORIA DE LA EDUCACIÓN EN COLOMBIA Y VENEZUELA)
}

\author{
Dr. José Pascual Mora García ${ }^{\mathbf{1}}$ \\ Universidad de Los Andes -Venezuela \\ Grupo de Investigación HISULA \\ pascualmoraster@gmail.com
}

\begin{abstract}
Recepción: 30/05/2008
Evaluación: 10/09/2009

Aceptación: 18/09/2009

Artículo de Revisión
\end{abstract}

\section{RESUMEN}

Este texto es el resultado de un estudio sobre la historia de la Universidad de Caracas, específicamente analizando el caso del Pbro Dr. Baltasar de los Reyes Marrero, quien era catedrático de Filosofía para seglares en 1788-1789. En el mismo se expone la crisis de paradigmas caracterizada por la escolástica y tomismo decadente y el paradigma emergente del método científico fundado en la física de Newton. Del mismo modo se hace un análisis comparativo con la mentalidad ilustrada en la Nueva Granada.

Palabras claves: Paradigma, Historia de la Universidad, Mentalidad Educativa.

\footnotetext{
1 Doctor en Historia y Doctor en Pedagogía (URV-Tarragona-España, 2009). Profesor de la Universidad de los Andes-Táchira, Categoría Asociado, Investigador reconocido por FONACIT- ONCTI- 2009 y miembro del grupo de investigación Historia y Prospectiva de la Universidad Latinoamericana HISULA. Vicepresidente de la Sociedad de Historia de la Educación Latinoamericana SHELA (2007-2011) elegido en Guadalajara, Estado de JaliscoMéxico en octubre de 2007. Ha escrito 16 libros y capítulos de Libros, publicados en Colombia, España México y Venezuela.
} 


\title{
BALTASAR DE LOS REYES MARRERO (1752-1809): FIRST PROFESSOR OF THE MODERN PHYSICS TEACHING AT CARACAS UNIVERSITY (LAST DECADES OF THE 18TH CENTURY). AN EDUCATION HISTORY CONNECTED BETWEEN COLOMBIA AND VENEZUELA
}

\author{
Dr. José Pascual Mora García \\ Universidad de Los Andes -Venezuela \\ HISULA Research Group \\ pascualmoraster@gmail.com
}

\begin{abstract}
This is the result from a study about The Caracas's University history, analyzing Pbro Dr. Baltasar de los Reyes Marrero's case, who was a Philosophy Professor in 1788-1789. It also shows the crisis of paradigms, featured by the Scholastic and decaying Thomism, and the emergent paradigm of the scientific method found in Newton's Physics. At the same, it makes comparative analysis with the illustrated mentality of New Granada.
\end{abstract}

Key words: paradigm, educational mentality, university history. 


\section{INTRODUCCIÓN}

\section{El tiempo histórico de la Ilustración en la antigua Universidad de Caracas ${ }^{2}$}

La presencia de un nuevo paradigma educativo no significa el desplazamiento radical del anterior; de hecho, podemos hablar de coexistencia de paradigmas: uno emergente y otro decadente. ${ }^{3}$ La Ilustración representa el paradigma emergente, en sus principios y acción está el germen de una nueva racionalidad que tiene por cimiento las leyes y las luces; sin embargo, la oficialidad no cedió el paradigma dominante sin antes someter a juicios y prisión a quienes se inspiraron en las ideas emergentes para introducir cambios en la universidad dieciochesca. ${ }^{4}$ Las luces representan el saber, el conocimiento y por ende la educación como centro de la vida social. En España y sus colonias "en el momento en que Carlos III sube al trono, la educación en los dominios de la Corona no se consideraba como un servicio público. En la organización y distribución de las escuelas y colegios predomina la mayor anarquía. El Monarca y sus ministros, acorde con su política educativa se proponen intervenir y lo hacen. Pero no se pretendía ni interesaba, apartar totalmente a la Iglesia, podemos comprobar cómo a pesar de los intentos secularizadores efectuados por las corporaciones locales, siguen los religiosos presidiendo los centros benéficos y educativos. Durante la ilustración, en el período

2 Este trabajo es un adelanto de la investigación: Análisis Bibliométrico de las Comunidades Discursivas de Historia de la Educación en Colombia, estudio de caso: RUDECOLOMBIA (1996-2008), con anuencia del CDCHT- ULA, Código: NUTA-H-280-08-06. El mismo forma parte de una línea de investigación iniciada en el año 2000, y avalada institucionalmente por el Consejo de Desarrollo Científico Humanístico y Tecnológico (CDCHT) de la Universidad de Los Andes con el proyecto Análisis Bibliométrico de las Comunidades Discursivas de Historia de la Educación en América Latina. Estudio de Caso: Venezuela. Código: NUTA-H170-02-06-B. Actualmente continuamos la temática pero vinculada al caso: RUDECOLOMBIA, proyecto de investigación NUTA-H-280-08-06-B, aprobado por el Directorio del CDCHT en la reunión 05-08 de fecha 03/12/08. Este proceso ha tendido importantes antecedentes, que ha merecido el ser invitado como investigador asociado del Grupo de Investigación HISULA (RUDECOLOMBIA), CADE- UPTC-Tunja; igualmente, como arbitro y miembro del Consejo Editorial de la Revista Historia de la Educación Latinoamericana. Desde el año 2007 formo parte de la Junta Directiva de la Sociedad de Historia de la Educación Latinoamericana, para el periodo 2007-2011, en condición de Vicepresidente, lo cual me obliga a profundizar en el estado del arte de los países que forman parte de la corporación académica, en este caso de Colombia. Como Profesor invitado al Doctorado de Ciencias de la Educación CADE- Tunja he participado en seminarios y conferencias. Incluso ha tenido como resultado actual la presentación de mi tesis doctoral en la Universidad Rovira Virgili, Tarragona-España (julio 2009), intitulada: Comunidades Discursivas de Historia de la Educación en América Latina. Defendida con mención honorífica Excelente Cum Laude por unanimidad.

3 Aquí seguimos el concepto de paradigma desarrollado por Kuhn. Cfr. KUHN, Thomas. (1971): La Estructura de las Revoluciones Cientificas. México. FCE.

4 Cfr. MORA GARCÍA, José Pascual. (1996): "De Carlos III a Simón Rodríguez, tres momentos de polémicas para los historiógrafos de la educación de Venezuela y Colombia", en MORA GARCÍA, José Pascual. (1996): Del fin de la historia a la postmodernidad. San Cristóbal. Ediciones de la Universidad de Los Andes-Táchira. Para un estudio más acabado al respecto puede verse MORA GARCÍA, José Pascual. (2004): La dama, el cura y el maestro. Historia social de las mentalidades y de la educación en la vicaría foránea de La Grita y región andina venezolana, Mérida, Consejo de Publicaciones de la Universidad de Los Andes. 
borbónico, empieza a acelerarse el proceso secularizador de prácticamente todas las áreas del Estado, afectando indudablemente a la Enseñanza como pilar fundamental en la reproducción ideológica." ${ }^{5}$ El Estado español reordena las políticas acerca de la economía y la administración, pues el objeto era transformar los reinos de ultramar, en particular, los reinos de Indias en un verdadero imperio económico. Eso implicaba no sólo modificar los impuestos sino aumentar la producción económica mediante la introducción de cambios técnicos en la producción, y eso significaba modificar los criterios acerca de la educación.

Los voceros de la Ilustración española, desde Benito Jerónimo Feijoo, Cabarrús, Gaspar de Jovellanos, Pedro de Campomanes, Fernández Navarrete, Cadalso, hasta el "privado" de Carlos III, Floridablanca; todos coinciden en ser defensores de la educación en las "artes útiles" como principio de prosperidad social y responsabilidad administrativa del Estado. La atmósfera mental que buscaba cambios substantivos en la educación española se debe en parte a la presencia de este grupo de filósofos, entre los que no debe faltar José de Viera y Clavijo. ${ }^{6}$ Todos rechazaban el modelo de talante medieval; Cabarrús en sus cartas a Gaspar de Jovellanos se quejaba de la educación que se impartía en España calificándola de "tétrica hipocresía monacal."

El aporte más importante durante la etapa de la Ilustración fue el surgimiento de la "secularización de la educación" y la ruptura epistemológica del paradigma educativo. Sin embargo, debemos advertir que el "espíritu" ilustrado no evolucionó linealmente; al respecto nos aclara Juan Manuel Santana que al subir Carlos IV al trono en 1788 se genera en España una triple división del pensamiento: antirreformistas, ilustrados o reformistas y los críticos o pre-liberales que en algunos casos, lejos de marcar, avances retrogradaron el proyecto ilustrado. Incluso algunos ilustrados devinieron en contrarrevolucionarios, como el caso del obispo Pedro Días (sic) de Valdés "quien pasa de heraldo de la educación ilustrada a partir de las parroquias, a delator de la introducción de las nuevas ideas entre los estudiantes de Cervera."9 Algunos hombres

\footnotetext{
5 SANTANA, Juan Manuel y MONZÓN, María Eugenia. (1994): La Iglesia en la Instrucción Pública Canaria del siglo XVIII, p. 523.

6 Viera y Clavijo es considerado el más genuino representante de la filosofía Ilustrada en Canarias, como bien apuntan Juan Manuel Santana y María Monzón, pues "dada la penuria existente en la reflexión pedagógica de aquella época, los Memoriales del Síndico Personero, periódico manuscrito hecho por el más destacado personaje canario de la ilustración, José Viera y Clavijo (clérigo, enciclopedista y librepensador), se convierten en un documento de extraordinario valor histórico-pedagógico. SANTANA, Juan Manuel y MONZÓN, María Eugenia. (1994) Op., cit, p. 171.

7 "Oh amigo mío. No sé si el pecho de Vuestra Merced participa de la indignación vigorosa del mío al ver estos rebaños de muchachos conducidos en nuestras calles por un escapulario armado de su caña." Cfr. CABARRUS. (1926). "Cartas del señor don Gaspar de Jovellanos.". en Epistolario Español, Colección de Cartas de Españoles ilustres antiguos y modernos recogida y ordenada con notas de aclaraciones históricas, críticas y biográficas por Eugenio de Ochoa.

8 SOTO ARANGO, Diana, PUIG-SAMPER, Miguel Ángel y ARBOLEDA, Luis Carlos. (1995): La Ilustración en América Colonial. Madrid, Ediciones Doce Calles, COLCIENCIAS, CSIC.

9 SANTANA, Juan Manuel. (1999): "Impulso de la Ilustración Española en el reinado de Carlos IV," en Cuadernos del Sur, $\mathrm{N}^{\circ}$ 28. Argentina, pp. 275-276.
} 
del gobierno que representaban el brazo ilustrado fueron perseguidos y encarcelados: "Cabarrús fue denunciado ante la Inquisición y encarcelado, Jovellanos fue desterrado a Asturias y Campomanes fue cesado en la presidencia del Consejo de Castilla (...) Floridablanca (...) fue cesado el 28 de febrero de 1792 (...) Aranda fue sustituido por Godoy. " 10 En el fondo el problema era que las ideas de la Revolución Francesa representaban una amenaza para la monarquía. No podemos centrar el desplazamiento del paradigma aristotélico-tomista en las políticas educativas de Carlos III, ni la retrogradación del modelo educativo ilustrado con el ascenso de Carlos IV. Sería olvidar que el "espíritu" de las Luces se impuso poco a poco logrando transformarse en una weltanschauung que impactaría la cultura occidental.

La lucha ideológica entre los rancios criterios inspirados en el Paradigma Aristotélico-Tomista y el emergente Paradigma Educativo de la Ilustración no sólo fue de palabra, sino que tuvo implicaciones de jure, como el juicio a algunos adelantados en los modernos métodos científicos y educativos; el padre A. Valberde, (sic) en 1770, señalaba que "1) La filosofía de Aristóteles, ni para el conocimiento de la naturaleza, ni para tratar la sagrada teología es útil, sino perniciosa; y 2) que Santo Tomás floreció en los siglos de la ignorancia". ${ }^{11}$ Pero quizá el caso que tuvo mayor estelaridad fue el de Baltasar de los Reyes Marrero, profesor de la cátedra de Filosofía en la Real y Pontificia Universidad de Caracas, justamente porque "no enseñaba la filosofía de Aristóteles sino la moderna doctrina de Newton, Paracelso."12

Apreciaciones Metodológicas: El caso de Baltasar de los Reyes Marrero ha sido abordado por excelentes exponentes de la historia de la ciencia y de la Educación en Venezuela y Colombia, lo que nos presenta un compromiso al abordar el mismo. En particular recordamos los trabajos de Ildefonso Leal ${ }^{13}$ y Yajaira Freites, ${ }^{14}$ y un trabajo poco conocido de Luis Carlos Arboleda y Diana Soto Arango (1991), además de Remedios Ferrero Micó (2006) ${ }^{15}$ y Reinaldo Rojas (2009). ${ }^{16}$

${ }^{10}$ Ibídem, p. 281

11 FERNÁNDEZ HERES, Rafael. (1981): Memoria de cien años, Caracas, Ediciones del Ministerio de Educación, Tomo I, p. 47.

${ }^{12}$ Ibídem, p. 54.

13 A Ildefonso Leal, le debemos el mérito de rescatar el "Expediente del juicio entre don Baltasar Marrero y don Cayetano Montenegro sobre la expulsión de su hijo José Cayetano Montenegro de la clase de Filosofía que regentaba el primero, en la Real y Pontificia Universidad de Caracas, año 1790, reproducido en Boletín del Archivo Histórico de la UCV, 1984, No. 2:25-186.

${ }^{14}$ FREITES, Yajaira. (1995): Conocimiento y técnica en la Venezuela de la Ilustración: una aproximación, en La Ilustración en América Latina. SOTO ARANGO, Diana; PUIGSAMPER, Miguel, ARBOLEDA, Luis Carlos (eds.). Bibliografía Critica, Madrid, Consejo Superior de Investigaciones Científicas, Ediciones Doce Calles y Colciencias, pp. 141-161.

${ }_{15}$ El trabajo de la Remedios Ferrero es muy importante sobre todo en lo que atañe al estudio de la hacienda universitaria, que como bien apunta ha sido menos privilegiado. Cfr. FERRERO MICÓ, Remedios. (2006): La Universidad de Caracas y su implantación en el movimiento independentista, en Políticas universitarias en Latinoamérica: Historia y Perspectiva. SOTO ARANGO, Diana y LIMA JARDILINO, José Rubens (Directores), Sao Paulo, Ed. Emblema, pp. 12-30. Queremos reconocer que la Dra. Remedios Ferrero ha sido una de las investigadores 
Pero en nuestra aproximación buscamos establecer una actualización bibliométrica y un posible estudio comparativo-conectado entre el acontecer en Venezuela y la Nueva Granada para la época ilustrada. Por eso nuestro trabajo se ubica metodológicamente dentro de los llamados estudios bibliométricos. ${ }^{17}$ Como sabemos la bibliometría es una metodología que nos permite conocer el sentido teleológico de los productos: dónde van los artículos, cuáles son, cuál es su impacto, quién, cuándo, y dónde publica. Pero más que un estudio bibliométrico es un esfuerzo por realizar una metaevalución de las comunidades académicas a fin de presentar un estado del arte actualizado y que sirvan al dominio público; por eso es importante destacar estos resultados, porque beneficia no sólo a los investigadores noveles sino al conocimiento del avance científico, pertinencia de las investigaciones, y a la evaluación intersubjetiva; entre los elementos que podemos conocer están: a) la presencia de nuestras revistas en los índices internacionales; b) el índice de investigadores calificados que publican en el exterior y no publican en las revistas nacionales; c) mediciones de contribuciones de artículos científicos, por instituciones, publicados tanto a nivel mundial como nacionalmente; d) producción documental científica, distribuida por Centros de Investigación y promediada por investigadores; e) productividad de la investigación científica en una determinada área o disciplina, información que resultaría de vital importancia a la hora de realizar historias biográficas de las disciplinas en las universidades y en el país; f) el índice de dispersión de los artículos, el índice de inmediatez, media de vida de las revistas, variación en el número de referencias por artículos de revista en diversas disciplinas científicas, factor de impacto, revistas "rankeadas" por el número de citaciones a artículos de los dos años inmediatamente anteriores, en las referencias del corriente año, revistas jerarquizadas en sus respectivas áreas del conocimiento, o el listado alfabético de las revistas indicando en cuáles especialidades fueron publicadas. En fin podríamos seguir enumerando las bondades de ésta metodología pero creemos conveniente pasar a nuestro objetivo para tan sólo dar a conocer una de sus muchas aplicaciones. Arriesgamos algunos ejemplos vinculados al Análisis Bibliométrico descriptivo de manera que nos

más prominentes en estudiar la Universidad de Caracas en el periodo colonial. FERRERO MICÓ, Remedios. (1991) "Intentos de reorganización en la Universidad de Caracas al final del siglo XVIII." Actas del III Jornadas sobre Presencia Universitaria Española en América: la Universidad en la época Borbónica, Estudios de Historia Social y Económica de América," en Revista de la Universidad de Alcalá. $\mathrm{N}^{\mathrm{a}}$ 27, España, pp. 150-169. En esta misma dirección recomendamos: FERRERO MICÓ, Remedios. (2006) "Reformas en la Universidad de Caracas y conflictos de jurisdicción", en Reformas y Planes de Estudios de las Universidades de América y Europa, Córdoba (Argentina), T. I, pp. 121-145.

16 Recientemente Reinaldo Rojas acaba de presentar una ponencia: "De Baltasar de los Reyes Marrero a José María Vargas: origen de la Universidad Republicana", en el Panel de Educadores Latinoamericanos en el VII Congreso Internacional de la Sociedad de Historia de la Educación Latinoamericana. Sao Paulo, Brasil. Entre el 23 y 26 de agosto de 2009.

${ }^{17}$ Los especialistas en Análisis Bibliométrico (Curiel, 1999; Nicholas, 1978; y Stevens, 1953) consideran que es una ciencia cuantitativa de la cual se infieren consecuencias cualitativas, por eso dividen sus categorías básicas en dos: a) Bibliometría descriptiva, la cual se usa para productividad; y b) Bibliometría evaluativa, para contar la literatura utilizada en un tópico específico, materia o disciplina. Cfr. CURIEL, María Josefa. (1999): Contribución al Estudio de Algunos Elementos de Bibliometría, Caracas, UCV. En el mismo se realiza un análisis bibliométrico de la producción bibliográfica de las investigaciones del IVIC en la década 1980-1989. 
permitiera iniciar fundamentalmente una discusión epistemológica respecto a la investigación en la universidad venezolana y colombiana.

\section{La Mentalidad Ilustrada en la Nueva Granada}

En el Virreinato de la Nueva Granada el primer Plan educativo fue elaborado por Francisco Moreno y Escandón (1736-1792) en 1774, ${ }^{18}$ y a pesar de que fue una "clara imitación del verdadero método de estudiar del sacerdote portugués Luis Antonio Verney, conocido como el "Barbadiño", obra guía de las reformas educativas españolas"19 representa un quiebre en el tradicional paradigma educativo de herencia medieval, pues se aproxima al ideal de la Ilustración y a la diseminación de las ideas del empirismo y sensualismo. ${ }^{20}$

Las características del paradigma educativo emergente desplazaban el centro de gravedad de los conocimientos, al incentivar el desarrollo de los siguientes aspectos:

1. El impulso de la filosofía newtoniana basada en la observación, la experiencia, las matemáticas y la razón;

2. La separación de la razón del yugo de la revelación;

3. La defensa cerrada del regalismo en derecho; el rechazo al dogmatismo; y

4. La propuesta de una educación pública bajo la dirección y control estatal.

Poco a poco se estableció un planteamiento a favor de la comprensión racional en vez del memorismo estéril. Curiosamente, para esta época, en Bogotá se sucedía una discusión entre el profesor Manuel Santiago Vallecilla y el Rector del Colegio del Rosario, ${ }^{21}$ en la que la resistencia al cambio venía de parte del Rector Burgos, pues en un concurso de oposición se negó a aceptar que el "profesor Vallecilla (...), no sólo reafirmara su oposición al método escolástico, sino que también anunciaba que seguiría el método que había aprendido por el plan de estudios de Moreno y Escandón."”22 Aunque es importante señalar que la diseminación de los andamios mentales en la Nueva Granada se inicia con el sabio José Celestino Mutis, ${ }^{23}$ en especial, destacamos dos polémicas que hacen ver la inclinación hacia la ciencia experimental.

\footnotetext{
18 POSADA ALVAREZ, Rafael. (1992): La Filosofia Ilustrada y el plan educativo Moreno y Escandón (1774), Bogotá, Edición I Coloquio de Historiadores de la Educación de ColombiaUPN.

19 POSADA ALVAREZ, Rafael. (1992): Op. Cit, p. 59.

${ }^{20}$ Hay posiciones encontradas en los historiadores de Colombia, respecto a considerar el Plan Moreno y Escandón como muestra del paradigma ilustrado. JARAMILLO URIBE, Jaime. (1982): El pensamiento colombiano en el siglo XIX, Bogotá, Ediciones Temis.

${ }^{21}$ En la ciudad de Santa Fé de Bogotá en 1790 funcionaban cuatro instituciones de educación superior: la Universidad de Santo Tomás, la Universidad de San Nicolás de Bari, el Colegio Mayor del Rosario y el Colegio de San Bartolomé. SOTO ARANGO, Diana. (1992): Polémicas de los catedráticos universitarios en Santafé de Bogotá- siglo XVIII, Bogotá, Edición I Coloquio de Historiadores de la Educación de Colombia-UPN p. 176.

22 Ibídem, p. 177.

23 "Las matemáticas fueron, sin lugar a dudas, la base para el desarrollo del pensamiento ilustrado en el campo de la cultura de la Nueva Granada. A través de la cátedra, Mutis introdujo el pensamiento de Newton y Copérnico, suscitando debates dentro de los sectores más atrasados
} 
Una, la polémica con Sebastián José López Ruiz, por el descubrimiento de la quina en Bogotá, juicio que ganó el sabio Mutis. Yotra, la polémica con los dominicos respecto a la defensa del Sistema Copernicano en el Colegio del Rosario (1773), quienes lo acusaban de hereje. El juicio llegó hasta el Santo Oficio del Consejo de Castilla, pero nuevamente salió absuelto en 1775. Estos dos testimonios validan la lucha por la emergencia de una nueva mentalidad científica en la Nueva Granada. Sin embargo, el precedente de la enseñanza de la nueva física y matemática por parte de Mutis se remonta a 1762.

Francisco Antonio $\mathrm{Zea}^{24}$ fue otro de los nobles neogranadinos que tuvo una formación ilustrada, en especial, de su maestro José Félix de Restrepo (1760-1832), en el Real Colegio y Seminario de San Francisco de Asís de Popayán. Por cierto que tuvo como condiscípulo al sabio Francisco José de Caldas y al ideólogo de la independencia Camilo Torres. Es una muestra adicional de la emergencia de la mentalidad ilustrada en la Nueva Granada.

\section{Baltasar de los Reyes Marrero: El Clérigo Ilustrado}

Nació en Caracas el 6 de enero de 1752 y murió el 31 de mayo de 1809, de manera que estamos en el año bicentenario de su muerte. Sacerdote, catedrático, considerado el precursor de la física moderna en la Universidad de Caracas, Venezuela. Hijo de padres canarios, ${ }^{25}$ Domingo Marrero y Catalina de la Cruz Izquierdo. A los 12 años

\footnotetext{
de la sociedad, que se oponían al progreso y al desarrollo. No cabe duda de que en 1762, con la iniciación de la cátedra de matemáticas por parte de Mutis, se dio comienzo a una nueva etapa de la educación y la cultura neogranadinas que, unidas al plan de estudios presentado por el fiscal Francisco Moreno y Escandón, formaron un solo frente en la defensa de las ideas ilustradas, que haría avanzar la mentalidad de la juventud criolla del Nuevo reino." SOTO ARANGO, Diana. (2005): Mutis, el educador de la elite neogranadina, Bogotá, Edición Grupo ILAC, p. 76. Esta extraordinaria obra es contundente en el análisis por exponer la obra de Mutis como pensador ilustrado. Nos complace mucho que sea una investigación cuya línea de investigación la encontramos por primera vez en la tradición académica desde 1992. SOTO ARANGO, Diana y NEGRÍN FAJARDO, Olegario. (1992): "El debate sobre el sistema copernicano en la Nueva Granada en el siglo XVIII," Bogotá, I Congreso Iberoamericano de Docentes e Investigadores en Historia de la Educación Latinoamericana. p. 77

${ }^{24}$ SOTO ARANGO, Diana. (2000): Francisco Antonio Zea, un criollo ilustrado, España. Ediciones Doce Calles, COLCIENCIAS, RUDECOLOMBIA.

${ }^{25}$ La herencia canaria en Venezuela es notable, quizá sobre cualquier otro país de América Latina con excepción de Cuba y Puerto Rico en el siglo XVII y XVIII: "A partir de 1670 la emigración presenta un carácter masivo y familiar. Tras un siglo y medio de crecimiento se dan síntomas de crisis. La falta de salida del vidueño canario, un vino blanco de mesa, tras la emancipación de Portugal en 1640, cuyas colonias eran su mercado preferente, arrastra a numerosas familias isleñas, particularmente de Tenerife hacia tierras venezolanas y cubanas (...) La irrupción del cacao como producto de exportación y la pacificación y el control de Los Llanos favorecen la instalación definitiva de familias canarias en la región central de Venezuela. En la Caracas y La Guaira de fines del siglo XVII comienza a establecerse una importante colonia. Se calcula que representan entonces más de un $90 \%$ de los casamientos de inmigrantes blancos y un $16 \%$ del total, número que es mucho mayor si se tiene en cuenta que la gran mayoría se casaron y tuvieron hijos antes de emigrar." HERNÁNDEZ GONZÁLEZ, Manuel. (1999): La emigración canaria a América a través de la historia. Los canarios en la Venezuela colonial (1670-1810), Tenerife, Ediciones Universidad de La Laguna, p. 5 ss; HERNÁNDEZ GONZÁLEZ, Manuel. (1999): La emigración canaria a América entre el libre comercio y la emancipación (1765-1824), Tenerife, Ediciones Universidad de La Laguna.
} 
ingresó en el seminario de Santa Rosa como colegial porcionista y en la Universidad de Caracas se graduó de Maestro en Filosofía ${ }^{26}$ el 24 octubre de 1773, y de doctor en Teología en $1774 .^{27}$ Notable es resaltar que la influencia de la tendencia filosófica escotista más que del decadente tomismo abrió un camino en la formación filosófica de Baltasar de los Reyes, que propició su interés por la física moderna. La diferencia fundamental con respecto a la comprensión de Dios por parte de un escotista, y por parte de un tomista, establecen la separación substantiva. Para un tomista, la existencia de Dios es demostrable; mientras que para el escotista, Dios es Dios y no necesita ser demostrado. Esta diferencia, que pareciera sutil, fue determinante en el "atrévete a pensar" kantiano ${ }^{28}$ que dio origen al hombre de la Ilustración; de la cual Marrero, es un representante al atreverse a pensar la filosofía de la física desde Newton y no desde Aristóteles. En lo sucesivo el hombre no tendrá que preocuparse por la demostración matemática o científica de Dios; para un creyente, como lo fue Marrero, de herencia escotista Dios no necesita ser de razón, sino de palabra; es decir, si Dios es Dios entonces no necesita el auxilio del hombre.

Una profunda renovación académica se avecinaba en la Universidad de Caracas, ${ }^{29}$ la dependencia tomista de la oficialidad "chocaba con las nuevas corrientes filosóficas

\footnotetext{
${ }^{26}$ Cuando nace Baltasar de los Reyes Marrero, el pensamiento filosófico venezolano de herencia escotista manifestaba síntomas de madurez significativa reconocidas no sólo en Venezuela sino en España, como en efecto se puede evidenciar con la publicación de obras; entre ellos citamos a los siguientes: Agustín de Quevedo y Villegas, nacido en Coro-Venezuela, su obra Opera Theologica fue publicada en Sevilla en 1752; Tomás Valero, nativo de El Tocuyo-Actual Estado Lara-Venezuela, su obra Theologia Expositiva, publicada en 1755; y Juan Antonio Navarrete, nativo de Las Guamas-San Felipe-Venezuela, es prácticamente coetáneo con Marrero pues nació el 11 de enero de 1749. Su obra más importante fue Arca de Letras y Teatro Universal. Las tendencias filosóficas más importantes en la Universidad de Caracas tiene dos orientes; uno, caracterizado por el escotismo de Alfonso Briceño, Quevedo y Villegas y Tomás Valero. Y otra, la herencia tomista delineada por dos caraqueños: Urbina y Suárez. El antecedente más importante es esta dirección es la obra de Alfonso Briceño (1590-1668), quien era chileno pero su obra fue escrita en Trujillo-Venezuela, y "debe ser considerado el precursor del pensamiento filosófico venezolano. En el siglo XVII no hay aquí, en toda la América, ni tal vez tampoco en Europa, quien lo aventaje." p. 18; GARCÍA BACCA, Juan David. (1954): Antología del pensamiento filosófico venezolano, Caracas, Ediciones de la Presidencia de la República.

27 Recibió en Barquisimeto del obispo Martí las órdenes en marzo de 1779, Ejerció la cátedra de Menores (1774-1775), y estudio también medicina con el fundador de la cátedra el Dr. Lorenzo Campins (1774-1775)

${ }^{28}$ La frase de Emmanuel Kant, el conocido Sapere aude, atrévete a pensar por ti mismo, se convierte en la esencia del hombre ilustrado: "La ilustración es la salida del hombre de su minoría de edad. El mismo es culpable de ella. La minoría de edad estriba en la incapacidad de servirse del propio entendimiento, sin la dirección de otro. Uno mismo es culpable de esta minoría de edad cuando la causa de ella no yace en un defecto del entendimiento, sino en la falta de decisión y ánimo para servirse con independencia de él, sin la conducción de otro. ¡Sapere aude! ¡Ten valor de servirte de tu propio entendimiento! He aquí la divisa de la ilustración." KANT, Manuel. (1987): Filosofia de la Historia, Madrid, Tecnos, 1987, pp. 3-23. ${ }^{29}$ La Universidad de Caracas (Real y Pontificia Universidad de Caracas, 1721) seguía el modelo escolástico con cuatro facultades: Teología, Derecho (civil y canónico), Medicina y Artes. Con el nombre de ésta última se designaba a las siete artes liberales: el Trivium (Gramática, Retórica y Lógica) y el Cuatrivium (Aritmética, Música, Geometría y Astronomía). El título de Bachiller en Artes era preparatorio para los demás. Más adelante se obtenían los grados de
} 
y teológicas que defendían los seglares, tanto laicos como eclesiásticos, que ocupaban algunas de sus cátedras y que alternativamente accedían a su rectorado. A medida que avanza el siglo y con él la penetración de las nuevas ideas, como acontece en La Habana y Caracas, éstos tratan de incorporar a sus estudios nuevos planteamientos, lo que convierte a la Universidad en un escenario de constantes controversias que contrasta con la mayor penetración y hegemonía del catolicismo ilustrado en los Seminarios Conciliares. "Ese carácter mixto, aunque con control dominico en numerosas cátedras y cargos, como el de cancelario, será objeto de una viva controversia y de pugnas como la acontecida con Baltasar Marrero en la Universidad" 30 caraqueña al incorporar a su enseñanza el racionalismo. Lo mismo sucedía con la medicina, el sector universitario más permeable a las nuevas ideas." ${ }^{31}$

No cabe duda que Carlos III fue un adelantado de la Ilustración, incluso la autonomía de la universidad fue una decisión que podríamos decir coloca la Universidad de Caracas casi por decreto en la etapa Ilustrada; pues en 1784, el Monarca dispuso que era potestad del claustro elegir al Rector. La Real cédula fechada en San Lorenzo a 4-X-1784, dispone el principio de autonomía universitaria, pues el Rey Carlos III estableció que el Rector fuera elegido por el Claustro Pleno y no por el Obispo, como se estilaba desde que se fundó la Universidad en 1721.

\section{Cuadro 1}

Organigrama de la Universidad de Caracas (1785-1791) ${ }^{32}$ (Rectores y Vicerrectores)

\begin{tabular}{|c|c|c|}
\hline & Rectores & Vicerrectores \\
\hline $1785-1787$ & Dr. José Domingo Blanco & Dr. Juan Agustín de la Torre \\
$1787-1789$ & Pbro. Dr. José Ignacio Moreno & Pbro Dr. Domingo Briceño \\
$1789-1791$ & Dr. Juan Agustín de la Torre & Maestro Juan Luis Escalona \\
\hline
\end{tabular}

Bachiller, Maestro (licenciado) y Doctor en Teología, Derecho y Medicina. Sin embargo, la fundación de la cátedra de Medicina atraviesa por tres momentos infructuosos entre 1727 y 1763, cuando definitivamente se instala por el médico mallorquín don Lorenzo Campins y Ballester. Cfr. LEAL, Ildefonso. (1968): Historia de la Universidad de Caracas (1721.1827). Caracas, Ediciones Biblioteca de la Universidad Central de Venezuela, pp. 212 y 213.

${ }^{30}$ Ibídem., p. 298

31 HERNÁNDEZ GONZÁLEZ, Manuel. (2008): "Evolución histórica de las Universidades Dominicanas (1650-1795)" en Revista Heurística, revista digital de historia de la educación en Venezuela. $\mathrm{N}^{\circ} 10$, julio- diciembre. Mérida, Universidad de Los Andes. Plataforma institucional: www.saber.ula.ve. p. 45.

32 LEAL, Ildefonso. (1963): Historia de la Universidad de Caracas (17211827). Caracas. Ediciones de la Biblioteca de la Universidad Central de Venezuela, p. 401.

\begin{tabular}{l|l} 
Rhela. Vol. 13. Año 2009, pp. $148-165$ \\
\hline &
\end{tabular}


El Cancelario ${ }^{33}$ fijaba la fecha del grado, y ese día se trasladaban dos doctores a la morada del graduado. Luego se iniciaba el paseo en donde se exhibía por la ciudad al nuevo doctor. Consistía en una procesión precedida de una caballería, al frente marchaban los músicos. El nuevo doctor llevaba los hombros cubiertos con una muceta y descubierta la cabeza, y el bonete que se le destinaba iba colgado de una vara en manos de uno de los doctores acompañantes.

Cuadro 2

Cancelarios (1778-1794) ${ }^{34}$

\begin{tabular}{|c|c|}
\hline & Cancelarios \\
\hline $1778-1788$ & Pbro Dr. Lorenzo Fernández de León \\
$1788-1791$ & Pbro Dr. Domingo Hermoso Mendoza \\
$1791-1794$ & Pbro. Dr. Luis Antonio Méndez Quiñones \\
\hline
\end{tabular}

Cuadro 3

Catedrático: Latinidad de Menores (1773-1775) ${ }^{35}$

\begin{tabular}{|c|c|}
\hline & $\begin{array}{c}\text { Catedrático: } \\
\text { Latinidad de Menores }\end{array}$ \\
\hline 1773 & Pbro. Dr. Ignacio Moreno \\
1775 & Pbro. Dr. Baltasar de los Reyes Marrero \\
\hline
\end{tabular}

Los estudios en la Universidad de Caracas requerían de cursos según el nivel; a nivel de Bachillerato: se requería 3 años de Arte o Filosofia, 4 en Teología, 4 en Medicina, 5 en Jurisprudencia civil, y 5 en Jurisprudencia canónica. La Licenciatura requería de dos años como profesor adjunto. La Licenciatura en Leyes tenía que superar un examen sobre el Digesto Viejo y el Código de Justiniano. En Cánones sobre las Decretales de Gregorio Nono y el Decreto; en Teología sobre los 4 primeros libros del Maestro de las sentencias; en Medicina sobre los aforismos de Hipócrates y la Física de Aristóteles; y

\footnotetext{
${ }^{33}$ La Universidad de Caracas había nacido Real (1721) y Pontificia (1722), fundada conforme a la Universidad de Santo Domingo y con sus privilegios, pero su legislación no se inspiró en ella ni en la de La Habana. Recibe influjos de Alcalá y Salamanca, pero también de Lima, y de hecho su legislación influyó en las dos universidades antillanas, mientras que la figura del Cancelario de Caracas sigue en todo las prácticas de Salamanca. Aunque imitó las prácticas de Santo Domingo, a la hora de redactar sus Constituciones obtuvo un resultado distinto al de Santo Domingo y La Habana. Cfr. ALONSO MARAÑóN, Pedro Manuel, (2006): "La vinculación de la Universidad de Alcalá con las universidades hispanoamericanas: perspectiva histórica y proyección," en Revista Heurística, revista digital de historia de la educación en Venezuela. $\mathrm{N}^{\circ}$ 5, enero junio. Mérida, Universidad de Los Andes. Plataforma institucional: www.saber.ula.ve.

${ }^{34}$ Ibídem, p. 403

35 Ibídem, p. 404
} 
José Pascual Mora García

en Filosofía por los tratados Lógica, Física, de Coelo, de Generatione, de Anima, y la Metafísica de Aristóteles. El cancelario era el encargado de citar al candidato.

Cuadro 4

Filosofía de Seglares (1776-1791) ${ }^{36}$

\begin{tabular}{|c|c|}
\hline & Filosofía de Seglares \\
\hline 1776 & Pbro. Dr. José Ignacio Moreno \\
1788 & Pbro. Dr. Baltasar de los Reyes Marrero \\
1791 & Pbro. Dr. Francisco Antonio Pimentel \\
\hline
\end{tabular}

Entre los discípulos más destacados de Baltasar de los Reyes Marrero citamos a Rafael Escalona Arguinzones (1773-1853), ${ }^{37}$ Francisco Pimentel, y Alejandro de Echezuría. Rafael Escalona Arguinzones regentó la cátedra de Filosofía en julio de 1797, siguiendo las instrucciones de su maestro y tiene el mérito de sobreponerse a la rancia tradición tomista y escotista al continuar la enseñanza de la filosofía y física moderna. Fue profesor de filosofía de Andrés Bello; herencia que podemos constatar al analizar la Filosofía del Entendimiento ${ }^{38}$ en donde nos dejó un perfil de las tradiciones filosóficas más importante de su tiempo, entre ellas la herencia empirista. Es posible que el haber sido secretario privado de Jeremías Bentham le haya llegado la influencia del empirismo inglés de David Hume y John Locke, pues Bentham es considerado el fundador del positivismo utilitarista de reigrambre empirista. Por cierto que la obra de Bentham también ejerció influencia en la Universidad durante la etapa de la Gran Colombia cuando fue adoptado su texto Principios de Moral y "Legislación (1789) en los estudios de Derecho"39. Así pues, Bello, nos presenta una referencia de la tradición empirista siguiendo a David Hume, Condillac, Desutt de Tracy, y el mismo Locke. La obra Filosofia del Entendimiento fue preparada como libro de texto para los estudiantes de Chile pero puede ser considerada una de las obras maestras de la filosofía venezolana de todos los tiempos.

\footnotetext{
${ }^{36}$ Ibídem, p. 405

${ }^{37}$ Hijo de Juan Luis de Escalona y de Francisca Arguinzones y Oviedo, emparentado con José de Oviedo y Baños y hermano de Juan de Escalona. Se destacó en Derecho Público y doctorado en la doctrina canónica en la Real y Pontificia Universidad de Caracas. Fue profesor de latín; alcanzó prestigio por su dominio.

${ }^{38}$ BELLO, Andrés. (1951): Filosofia del Entendimiento y otros escritos filosóficos. Caracas. Ediciones Ministerio de Educación. Si tuviéramos que juzgar la herencia de Baltasar de los Reyes Marrero tendríamos que revisar esta obra de Andrés Bello. Nos enseña la historia de las mentalidades que la obra de los maestros es posible seguirla a partir de los apuntes de sus discípulos, aunque sean indirectos. Lo propio sucedió con Carlos Marx quien no fue discípulo directo de G. W. F. Hegel sino de sus discípulos del Club de los Doctores de Berlín (Bruno y Otto Bauer, Max Stirner, Moses Hess), y logró interpretar de manera magistral la dialéctica hegeliana aplicándola como método a su propuesta filosófica. La confesión de Bello respecto a sus formadores la expresa así: "e dado cuenta de mis principios, de mi plan y de mi objeto y he reconocido, como era justo, mis obligaciones a los que me han precedido." Cita citada por GARCÍA BACCA, Juan David. (1949): "Prólogo a Filosofía del Entendimiento." Caracas. Ediciones Ministerio de Educación, p. XIV.

${ }^{39}$ Ibídem, p. 405
} 
La confesión de Bello respecto a sus formadores la expresa así: "E dado cuenta de mis principios, de mi plan y de mi objeto, y he reconocido, como era justo, mis obligaciones a los que me han precedido. Señalo rumbos no explorados, y es probable que no siempre haya hecho en ellos las observaciones necesarias para deducir generalidades exactas. Si todo lo que propongo de nuevo no pareciera aceptable, mi ambición, quedará satisfecha con que alguna parte lo sea, y contribuya a la mejora de un ramo de la enseñanza, que no es ciertamente el más lucido, pero es uno de los más necesarios." ${ }^{40}$

En su lucha contra la escolástica, Baltasar de los Reyes Marrero, expuso su curso de filosofía natural y lógica a impartir de 1788, igualmente su curso de nociones de álgebra, aritmética y geometría por considerarlas «...indispensables y necesarias para la verdadera inteligencia de la Física, y aún de la misma Sagrada Teología...» También dio a conocer los principios físico-matemáticos de Newton, ${ }^{41}$ las leyes de Kepler, el sistema de Copérnico, las teorías químicas de Duvy, Stahl y Lavoiser, y Cosh. Así como las opiniones científicas de Benjamín Franklin y Volta, acerca de la electricidad. En el campo propiamente filosófico alentó a los alumnos al estudio de las doctrinas de Locke, Condillac, Leibnitz, Wolff, Bacon y Lamarck.

La resistencia al cambio aquí no venía de la oficialidad sino de uno de los padres o representantes, específicamente del Dr. Cayetano Montenegro. El rector Juan Agustín de la Torre, del bienio 1789-1791, se pronuncia en el juicio a favor de Baltasar de los Reyes Marrero alegando que "ninguna nación ha hecho progresos de consecuencia por las armas, por las artes, agricultura y comercio, hasta que se ha entregado al indispensable cultivo de las ciencias»». ${ }^{42}$ Respuesta que estaba en consonancia con su criterio acerca de los cambios que debía experimentar la educación en Caracas, y toda Venezuela.

Juan Agustín de la Torre con su discurso económico, y Miguel José Sanz con su discurso jurídico marcó una pauta con respecto a las políticas educativas en la Caracas de fines del siglo XVIII. El primero, fue el encendido Discurso Económico, Amor a las Letras con relación a la Agricultura y Comercio, dirigido al Real Consulado en Abril de 1790 , en el que señala que:

Los ingenios no son regularmente comunes y generales para todas las ciencias, las inclinaciones de los hombres son particulares y adictas a determinadas pretensiones, nacen con cierta disposición proporcionada para

\footnotetext{
40 Ibídem., p. XVI.

41 La primera edición del Principia Matemática Philosophiae Naturalis de Isaac Newton es de 1687. Sin embargo, la resistencia al cambio puede estimarse en la misma Europa ilustrada; pasaron 50 años antes que J. M. Voltaire la incorporara contra la opinión de casi todos los físicos franceses. Cfr. VOLTAIRE, Jean Marie. (1745): Eléments de Philosophie de Newton, citada por PARRA LEÓN, Caracciolo. (1989): Filosofía Universitaria Venezolana, Caracas, Ediciones de la Secretaría de la Universidad Central de Venezuela, (1 ra edición 1934, Caracas, Editorial Suramérica.)

42 FERNÁNDEZ HERES, Rafael. (1981): Op. Cit. p. 100, (Extracto del Discurso Económico del Dr. Juan Agustín de la Torre).
} 
José Pascual Mora García

ser útiles en la vida civil en este o aquel destino: y desde que les raya la luz de la razón, comienzan a descubrir con sus naturales impulsos el objeto a que deben ser dedicados. Por esto una población como la de Caracas, capital de otras muchas provincias, debe tener copia de ministros de todas facultades para que halle su dilatada y estudiosa juventud los medios oportunos en que ejercitar su talento, porque si se les mezquina la instrucción privándolos de aquellos conocimientos adecuados a sus propensiones será precisarlos a que abracen los que les son repugnantes o enteramente contrarios a su genial condición. Lejos entonces de ser útiles a la sociedad, resultan gravosos a los pueblos, se entregarán a la vida holgazana, la autoridad de los magistrados no podrá reducirlos al trabajo y honestas ocupaciones, estarán en la república como los forzados en galeras; y por último, no sólo mirarán con grande odio cualquier ejercicio útil sino que también criarán tedio hasta de sus mismas personas. ${ }^{43}$

Y, el segundo, el Discurso en el Acto de Instalación de la Real Academia de Derecho público y español, en el que se apunta:

Así como la educación y ejemplo deciden el carácter del hombre, que aunque tenga en sí las raíces de la virtud moral, llega sin el apoyo de la razón ilustrada, a degradarse de su dignidad, abandonándose a sus torpes pasiones y apetitos. Si estudiara la ley de la naturaleza con sus relaciones civiles y políticas, hacia sí, hacia sus prójimos y hacia los soberanos o representantes del Estado, cumpliría sus funciones y destino, suavizaría sus costumbres, sacudiría preocupaciones vulgares, pensamientos altivos y otros vicios de presunción y soberbia que les envilecen tanto cuanto concibe de sí lo que no es, o lo que no conviene, haciendo consistir la verdadera gloria en vanidades $y$ aun en la misma ignorancia, de que provienen la turbación del orden público, las revoluciones y la ruina de los Estados. ${ }^{44}$

En ambos casos, se observa la influencia del siglo de las Luces, al pretender imponer el imperio de las Leyes y la Razón Ilustrada como principio fundamental de la sociedad; principios de fueron desarrollados en Francia por Montesquieu, Rousseau, Diderot, D'Alembert, y Condorcet. Pero también en los Ilustrados españoles: Cabarrús, Campomanes, Jovellanos, ${ }^{45}$ y Floridablanca.

\footnotetext{
43 DE LA TORRE, Juan Agustín. "Discurso Económico. Amor a las letras con relación a la agricultura y comercio." Citado por ANDRÉS LASHERAS, Jesús. (1997): «Educación y Estado en Venezuela: Historia de las bases ideológicas.» en Cuadernos de Postgrado No 16, Caracas-Venezuela, p. 46.

44 SANZ, Miguel José, "Discurso en el Acto de Instalación de la Real Academia de Derecho Público y Español" citado por FERNÁNDEZ HERES, Rafael. (1985): Vertientes Ideológicas de la Educación, Venezuela, Caracas, Ediciones Biblioteca de la Academia Nacional de la Historia, p. 44.

45 JOVELLANOS, Gaspar. Memorial sobre Instrucción pública., Citado por ANDRÉS LASHERAS, Jesús. (1997): Op. Cit, p. 45.
} 


\section{CONCLUSIONES}

En 1789, siendo Catedrático de Filosofía de Seglares, estalló una fuerte protesta contra él y se le acusó ante la Corte de Madrid de «infiel a Dios», que era prácticamente una forma de excomunión pública, al mismo tiempo que se le amenazó de hereje y de divulgador de «...máximas y doctrinas contrarias a las que el Rey tiene mandadas, de mal vasallo, sedicioso y rebelde...» Con estos cargos en su contra fue llevado el asunto al Consejo de Indias, donde se dio un veredicto en su contra, obligándosele a ceñirse al pie de la letra de los estatutos universitarios, y que las lecciones de álgebra, geometría y aritmética no eran obligatorias y solamente podían recibirlas aquellos estudiantes que voluntariamente lo expresaran. El Dr. Baltasar de los Reyes Marrero debió pagar 793 pesos de multa por las costas del litigio. Antes de conocerse en Caracas el veredicto de las autoridades peninsulares, Marrero renunció a la cátedra de Filosofía para servir el curato del puerto de La Guaira. El 9 de septiembre de 1800, cuando ejercía el cargo de tesorero de la catedral de Caracas, la Corona lo nombró Maestrescuela y Cancelario de la Universidad, empleo que desempeñó hasta su fallecimiento. ${ }^{46}$ Hay que destacar que el Rector Dr. Juan Agustín de la Torre "no solo defendió a Marrero sino que desplegó grandes esfuerzos para fundar una cátedra de Matemáticas." ${ }^{47}$ En vida nunca se le reconoció su aporte a la ciencia, tuvo que pagar el precio de todos los adelantados en la historia. Sólo con el advenimiento de la Universidad Republicana, el Claustro universitario emitió un acuerdo el 8 de octubre de 1827, que lo consagró como «Ilustre fundador de la Filosofía Moderna en Venezuela» y ordenó que los graduados «...lleven en sus títulos de bachiller, licenciado y maestro, la nota de discípulos de este inmortal Maestro...». ${ }^{48}$

Excuso La obra pionera de Baltasar de los Reyes Marrero es considerada como el principal antecedente de la fundación de la mecánica clásica en Venezuela, y al mismo tiempo precursor del positivismo utilitarista. En Venezuela estamos acostumbrados a dejarnos llevar por la idea diseminada en la obras de historia de la ciencia ${ }^{49}$ según la cual fue el sabio alemán Adolfo Erst quien trajo las ideas positivistas en 1860. Sin embargo, la idea positivista de que la ciencia debe estar ligada al método experimental ya estaba en las aulas de la antigua Universidad de Caracas a fines del siglo XVIII. Lo propio sucede en la Nueva Granada, tal como hemos podido constatar.

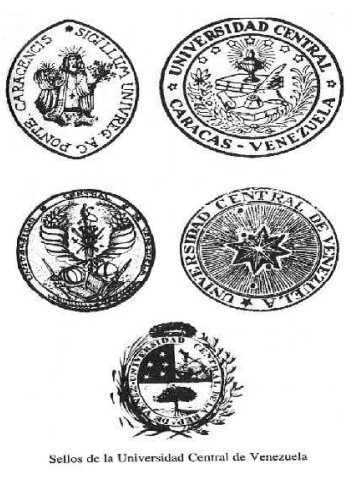

Ilustración 1: SELLOS

\footnotetext{
46 LEAL, Ildefonso. (1997): “Baltasar de los Reyes Marrero" en Diccionario de la Fundación Polar. Caracas. Ediciones de la Fundación Polar. Tomo III, p. 63.

${ }^{47}$ LEAL, Ildefonso. (1963): Historia de la Universidad de Caracas (17211827). Caracas. Ediciones de la Biblioteca de la Universidad Central de Venezuela, p. 266.

48 PARRA PÉREZ, Caraciolo. (1934) Op. Cit, p. 61. Cfr. Libro de Claustros, 1799-1843, f. 184. Archivo Universitario. La junta universitaria estuvo presidida por el Dr. José María Vargas, Rector.

49 GUERRERO, Luis Beltrán. (1956): “Introducción al Positivismo venezolano,” p 34.
} 


\section{SELECCIÓN BIBLIOGRÁFICA}

ALONSO MARAÑÓN, Pedro Manuel. (2006): "La vinculación de la Universidad de Alcalá con las universidades hispanoamericanas: perspectiva histórica y proyección." en Revista Heuristica, $\mathrm{N}^{\circ} 5$, enero junio. Mérida, Universidad de los Andes. Plataforma institucional.

BELLO, Andrés. (1951): Filosofia del Entendimiento y otros escritos filosóficos. Caracas. Ediciones Ministerio de Educación.

CURIEL, María Josefa (1999) Contribución al Estudio de Algunos Elementos de Bibliometría, Caracas, UCV.

DE LA TORRE, Juan Agustín. (1997): "Discurso Económico. Amor a las letras con relación a la agricultura y comercio." Citado por ANDRÉS LASHERAS, Jesús. Educación y Estado en Venezuela: Historia de las bases ideológicas. Cuadernos de Postgrado. (16). Caracas-Venezuela, p. 46

FERNÁNDEZ HERES, Rafael. (1981): Memoria de cien años, Caracas, Ediciones del Ministerio de Educación. Tomo I. p. 47.

FERRERO MICÓ, Remedios. (2006): La Universidad de Caracas y su implantación en el movimiento independentista, en Políticas universitarias en Latinoamérica: Historia y Perspectiva, SOTO ARANGO, Diana y LIMA JARDILINO, José Rubens (Directores), Sao Paulo, Ed. Emblema, pp. 12-30.

FREITES, Yajaira. (1995): Conocimiento y técnica en la Venezuela de la Ilustración: una aproximación, en SOTO ARANGO, Diana; PUIG-SAMPER, Miguel, ARBOLEDA, Luis Carlos (eds.). La Ilustración en América Latina, Bibliografía Critica, Madrid, Consejo Superior de Investigaciones Científicas, Ediciones Doce Calles y Colciencias, pp. 141-161.

GARCÍA BACCA, Juan David. (1954): Antología del pensamiento filosófico venezolano, Caracas, Ediciones de la Presidencia de la República.

GUERRERO, Luis Beltràn. (1956): "Introducción al positivismo venezolano", en A.V. Historia de la Cultura en Venezuela, Caracas, UCV.

HERNÁNDEZ GONZÁLEZ, Manuel. (2008): "Evolución histórica de las Universidades Dominicanas (1650-1795)" en Revista Heurística $\mathrm{N}^{\circ} 10$, julio- diciembre. Mérida, Universidad de Los Andes. Plataforma institucional.

HERNÁNDEZ GONZÁLEZ, Manuel. (1999): La emigración canaria a América a través de la historia. Los canarios en la Venezuela colonial (1670-1810). Tenerife, Ediciones Universidad de La Laguna.

HERNÁNDEZ GONZÁLEZ, Manuel. (1999): La emigración canaria a América entre el libre comercio y la emancipación (1765-1824), Tenerife, Ediciones Universidad de La Laguna. 
JOVELLANOS, Gaspar. (1997): Memorial sobre Instrucción pública. Citado por ANDRÉS en, LASHERAS, Jesús, Educación y Estado en Venezuela: Historia de las bases ideológicas. Cuadernos de Postgrado, Caracas-Venezuela.

KANT, Manuel. (1987): Filosofía de la Historia, Tecnos, Madrid.

KUHN, Thomas. (1971): La Estructura de las Revoluciones Científicas, México, FCE.

LEAL, Ildefonso. (1963): Historia de la Universidad de Caracas (1721-1827), Caracas, Ediciones de la Biblioteca de la Universidad Central de Venezuela, p. 401

(1997): "Baltasar de los Reyes Marrero" en Diccionario de la Fundación Polar, Caracas, Ediciones de la Fundación Polar.

MORA GARCÍA, José Pascual. (2004): La dama, el cura y el maestro. Historia social de las mentalidades y de la educación en la vicaría foránea de La Grita y región andina venezolana, Mérida, Consejo de Publicaciones de la Universidad de Los Andes.

(1996): Del fin de la historia a la postmodernidad, San Cristóbal, Ediciones de la Universidad de Los Andes-Táchira.

PARRA LEÓN, Caracciolo. (1989): Filosofía Universitaria Venezolana, Caracas, Ediciones de la Secretaría de la Universidad Central de Venezuela. (1ra edición 1934, Caracas. Editorial Suramérica.)

POSADA ALVAREZ, Rafael. (1992): La Filosofía Ilustrada y el plan educativo Moreno y Escandón (1774), Bogotá. Edición I Coloquio de Historiadores de la Educación de Colombia-UPN.

RODRÍGUEZ-SAN PEDRO BEZARES, Luis Enrique (Editor), Las universidades hispánicas de la monarquía de los Austrias al centralismo liberal. I. Siglos XVI-XVII, Salamanca.

SANTANA, Juan Manuel y MONZÓN, María Eugenia. (1993): La Iglesia en la Instrucción Pública Canaria del siglo XVIII, Gran Canarias: Cabildo Insular.

SANTANA, Juan Manuel. (1999): «Impulso de la Ilustración Española en el reinado de Carlos IV.,» en Cuadernos del Sur, $\mathrm{N}^{\circ} 28$. Argentina.

SANZ, Miguel José. "Discurso en el Acto de Instalación de la Real Academia de Derecho Público y Español” citado por FERNÁNDEZ HERES, Rafael (1985): Vertientes Ideológicas de la Educación en Venezuela., Caracas. Ediciones Biblioteca de la Academia Nacional de la Historia.

SOTO ARANGO, Diana. PUIG-SAMPER. Miguel Ángel y ARBOLEDA, Luis Carlos. (1995): La Ilustración en América colonial, Madrid, Ediciones Doce Calles, COLCIENCIAS, CSIC.

SOTO ARANGO, Diana. (1992): Polémicas de los catedráticos universitarios en Santafé 
José Pascual Mora García

de Bogotá- siglo XVIII, Bogotá. Edición I Coloquio de Historiadores de la Educación de Colombia-UPN.

SOTO ARANGO, Diana. (2005): Mutis, el educador de la elite neogranadina, Bogotá. Edición Grupo ILAC.

SOTO ARANGO, Diana y NEGRÍN FAJARDO, Olegario. (1992): El debate sobre el sistema copernicano en la Nueva Granada en el siglo XVIII, Bogotá. I Congreso Iberoamericano de Docentes e Investigadores en Historia de la Educación Latinoamericana.

SOTO ARANGO, Diana. (2000): Francisco Antonio Zea, un criollo ilustrado, España. Ediciones Doce Calles; COLCIENCIAS; RUDECOLOMBIA.

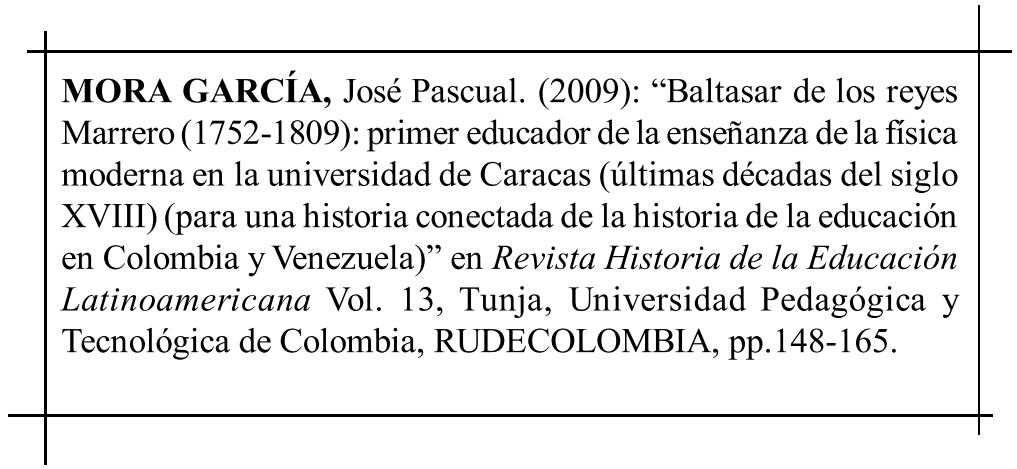

Rhela. Vol. 13. Año 2009, pp. 148 - 165 